\title{
THE GOLDEN SECTION IN THE NEXUS NETWORK JOURNAL
}

The Golden Section, commonly denoted by phi, or $\phi$, is almost certainly the most controversial subject in the interdisciplinary field served by the Nexus Network Journal. Its use as a design tool is not controversial. Indeed, it is hard to argue with the considerable potential for useful proportional relationships available to the designer, based on the mathematical properties of the Golden Section, whereby additive and multiplicative properties are so beautifully married because of the fact that $\phi^{2}=\phi+1$ (the square of the Golden Section is equal to the Golden Section plus Unity).

No, the Golden Section is controversial because of the large number of questionable claims about applications of the Golden Section to a wide range of objects, both natural and human-made. In the previous issue of the $N N J$, we presented a controversy over the conjectured use of the Golden Section in Palladio's Villa Emo (see "Palladio's Villa Emo: The Golden Proportion Hypothesis Rebutted" by Lionel March, and "Palladio's Villa Emo: The Golden Proportion Hypothesis Defended" by Rachel Fletcher in the NNJvol. 3 no. 2, Summer-Fall 2001). The current issue is dedicated completely to the Golden Section, and we hope to foster the kind of meaningful debate exemplified by these two articles from the last issue.

Any scholarly discussion involving the Golden Section in architecture should be grounded in an understanding of the evidence available in architectural literature. To this end, Marcus Frings offers "The Golden Section in Architectural Theory". Starting with the Ten Books of Vitruvius and ending with Le Corbusier's Modulor, this thoroughly researched essay reviews the appearance of the Golden Section - and lack thereof - in architectural writing. It provides a great service to those yearning for an accurate historical summary, and the reference list comprises a comprehensive compendium for further study.

The substantial role of the Golden Section in the climactic thirteenth book of Euclid's Elements is indisputable. Indeed, the "division in extreme and mean ratio" (the ancient Greek name for the Golden Section) is inherent in various proportional relationships involving the five Platonic solids, as explicitly shown by Euclid. Christopher Glass draws from this Euclidean tradition for his design of "The Pythagopod". This project for a residence is reminiscent of Buckminster Fuller's geodesic dome, but the clever interlocking of the five Platonic solids necessitates proportions based on irrational quantities important to classical mathematicians, namely $\sqrt{2}$ and $\phi$.

John Sharp provides the mathematics behind the facts and fiction concerning "Spirals and the Golden Section". This accessible and well-illustrated account describes the mathematics of Archimedean and logarithmic spirals and reviews various approximate spiral constructions based on geometric objects involving the Golden Section, including the widely celebrated golden rectangle spiral, based on squares adjoined to increasingly larger golden rectangles. The relationships between the approximate spirals and their true 
logarithmic spiral counterparts are detailed, and the commonly cited (but incorrect) association of the golden rectangle spiral to the Nautilus shell is thoroughly debunked.

The "metallic means" are the (positive) solutions to the family of quadratic equations,

$x^{2}-n x-1=0$, one mean for each positive integer $n$. The first metallic mean $(n=1)$ is the golden mean (Golden Section), and the next two are often called the silver mean and bronze mean, respectively. Because of the simplicity of this family of quadratic equations, the metallic means find various applications in science. "More True Applications of the Golden Number," by Dirk Huylebrouck and Patrick Labarque, provides some new applications of the Golden Section and its relatives, in the diverse contexts of colour theory, bicycle gears, and optimality problems involving the scaling of rectangles.

For the Geometer's Angle, Marcus the Marinite (aka Mark Reynolds) has drawn from his considerable portfolio of constructions pertaining to the Golden Section in order to offer "R-Tiles," a group of shapes, tiles, patterns, tessellations, and relationships that make up a unique geometric system based on $\sqrt{ } \phi$. Following the precedent he set in the last issue, Marcus is joined by a collaborator who offers a complementary essay for the Geometer's Angle. In "The Indefinite Dyad and the Golden Section: Uncovering Plato's Second Principle," Scott A. Olsen hypothesizes and outlines an interpretation explaining Plato's principle of the "Greater and Lesser" (Indefinite Dyad), together with the "Divided Line" construction, by means of a novel association to the Golden Section.

In the book review section, John Sharp provides a personal reading of a perceived dichotomy between mathematics and geometry in Richard Padovan's new philosophical analysis of proportion, Proportion: Science, Philosophy, Architecture. Padovan's important new work raises questions about the nature of proportion in architecture and design; while not dedicated solely to the Golden Section, his book certainly includes a great deal of material on it. The second review marks the first of a journal article in the NNJ, namely Nigel Reading's "Dynamical Symmetries: Mathematical Synthesis between Chaos Theory (Complexity), Fractal Geometry, and the Golden Mean," which appeared in the popular magazine Architectural Design and has become regularly cited by young architects and theorists. Michael J. Ostwald and Stephen R. Wassell argue that Reading's article is mathematically vague and often incorrect, more pseudo-mathematical and metaphysical than scientific, and, most importantly, essentially devoid of substance regarding architectural design methodology.

This is the first issue of the Nexus Network Journal dedicated to a particular subject, and it is my pleasure to have accepted Editor in Chief Kim Williams's invitation to take the lead on the editing. I would be delighted to receive any feedback about this issue, so please write or e-mail me with any comments, positive or negative.

Stephen R. Wassell, Guest Editor

6 NEXUS NETWORK JOURNAL - VOL. 4, NO. 1, 2002 